\title{
Harmonic enhancement of Gunn oscillations in GaN
}

\author{
C. Sevik*, D. E. Y1lmaz* and C. Bulutay* \\ *Department of Physics, Bilkent University, Bilkent, Ankara 06800, Turkey
}

\begin{abstract}
High field transport in wide bandgap semiconductors like GaN is of great technological importance. The negative differential mobility regime at high fields, under suitable conditions, can lead to millimeter-wave Gunn oscillations. Using extensive simulation based an ensemble Monte Carlo technique, the prospects of GaN Gunn diodes are theoretically investigated. The possibility of operating these Gunn diodes at their higher harmonic modes are explored. Main finding of this research is that the carrier dynamics in $\mathrm{GaN}$ can be tailored by an optimum choice of doping profile, temperature and bias conditions so that the efficiency of higher harmonic Gunn oscillations can be boosted.
\end{abstract}

\section{INTRODUCTION}

Gallium nitride with its high negative differential mobility threshold, measured [1] to be above $200 \mathrm{kVcm}^{-1}$, is an appealing material for high power millimeter-wave Gunn diodes. An ever-present objective is to increase the operating frequency of the Gunn diodes, and a promising approach is to operate them at their higher harmonic modes rather than their fundamental mode. In this work, our aim is to present a comprehensive ensemble Monte Carlo assessment of efficiency and harmonic enhancement in $n$-type GaN Gunn diodes, exploring the effects of including a doping notch/mesa, channel concentration, DC bias and the lattice temperature. We would like to extract the trends rather than quantitative aspects so that our results can shed light on the physics of milimeter wave Gunn oscillations based on other materials as well.

\section{STRUCTURAL DETAILS AND RESULTS}

In the types of GaN-based Gunn structures we study, the overall active region length is always kept constant at a value of $l_{a}=1.2 \mu \mathrm{m}$. This active region is sandwiched between the heavily doped $n^{+}$regions with $n^{+}=2 \times$ $10^{18} \mathrm{~cm}^{-3}$; the results were seen to be unaffected when this is increased to $1 \times 10^{19} \mathrm{~cm}^{-3}$. The active region is composed of an active channel with a doping of $n=$ $3 \times 10^{17} \mathrm{~cm}^{-3}$ (unless stated otherwise) and a lightly $n$-doped doping notch having $n^{-}=1 \times 10^{16} \mathrm{~cm}^{-3}$ or a doping mesa with $n^{+}=5 \times 10^{17} \mathrm{~cm}^{-3}$.

The quantity of primary interest, oscillator efficiency, is defined as $\eta=P_{\mathrm{AC}} / P_{\mathrm{DC}}$, where $P_{\mathrm{AC}}$ is the timeaverage generated $\mathrm{AC}$ power and $P_{\mathrm{DC}}$ is the dissipated DC power by the Gunn diode. First, the effect of the
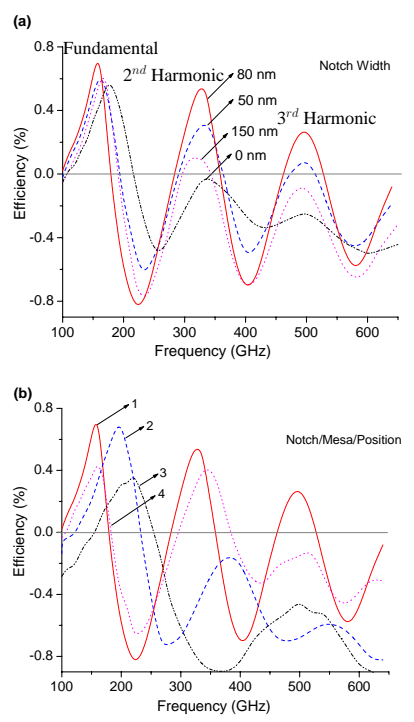

FIGURE 1. (a) Effect of different doping notch widths, (b) Comparison of the performance of four configuration made from the combinations of notch/mesa placed next to cathode and in the middle of the active region, while keeping the notch/mesa $=80 \mathrm{~nm}$. $1 \rightarrow$ notch next to cathode, $2 \rightarrow$ mesa next to cathode, $3 \rightarrow$ notch in the middle of the active region, $4 \rightarrow$ mesa in the middle of the active region.

doping notch width is investigated. It is observed that there is an optimum notch width, for our case, around a value of $80 \mathrm{~nm}$, at which the second harmonic efficiency approaches that of the fundamental mode value as seen in Fig. 1(a). Here, the fundamental mode corresponds to the frequency $v_{d, s a t} / l_{a}$ for a given active region length $l_{a}$, where $v_{d, s a t}$ is the saturation drift veloc- 
ity. In the $n^{\text {th }}$ harmonic mode, there exist $n$ Gunn domains travelling down the channel at each instant [3]. However, we would like to point out that the value of the optimum notch width is sensitive to the material parameters and our previous analysis with the bandstructure data of Ref. [4] resulted in a wider width [3]. On the other hand, a doping mesa rather than a notch was suggested to enhance the harmonic content of the current waveform [5, 6]. To elucidate this point, Fig. 1(b) compares the performance of four configurations made from the combinations of notch/mesa placed next to cathode and in the middle of the active region. It is seen that notch placed next to cathode (labelled as 1) performs the best among all. A source of curiosity regarding Fig. 1(b) is the substantial frequency shift for the notch placed in the middle (labelled as 3) and mesa placed next to the cathode (labelled as 2). Detailed investigation of these structures shows that for both cases almost half of the active region is inactive in the domain nucleation processes hence remarkably decreasing the transit time of the Gunn domains.
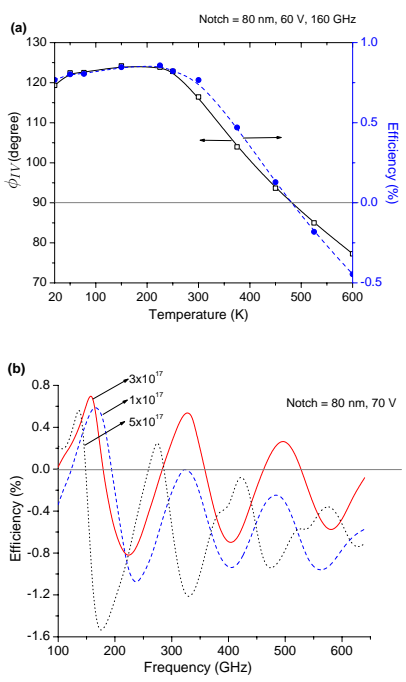

FIGURE 2. (a) Temperature versus phase angle between the current and voltage waveforms, and efficiency; $80 \mathrm{~nm}$-notch device at $60 \mathrm{~V}$ bias and its fundamental frequency $(160 \mathrm{GHz})$ is used. (b) RF conversion efficiency versus frequency for channel dopings; $80 \mathrm{~nm}$-notch device at $70 \mathrm{~V} \mathrm{DC}$ bias is used.

Next, the effects of DC bias is investigated. While changing the applied DC voltage across the Gunn diode we need to scale the RF amplitude accordingly not to lose the grounds for efficiency comparison. The applied DC voltage has to be above a critical value so that the device is biased in the negative differential mobility regime. According to our simulations (not shown), increasing bias has favorable effect on the fundamental and harmonic efficiencies. Another issue for high power Gunn diodes is thermal heating. At the expense of neglecting thermal gradient effects, we simply consider the uniform lattice temperature effects on the efficiency. Based on our results shown in Fig. 2(a), up to room temperature there is no sensible variation, whereas above the room temperature performance increasingly degrades becoming passive above $480 \mathrm{~K}$ in the case considered. The source of this degradation is not due to a reduction in the oscillation amplitudes but rather due to phase angle between the current and voltage waveforms shifting away from antiphase $\left(180^{\circ}\right)$ to orthogonal $\left(90^{\circ}\right)$ and to dissipative $\left(0^{0}\right)$ as the temperature increases. The increase in the lattice temperature does not significantly shift the frequency but diminishes the efficiency globally. Therefore, good heat sinking is required in these GaN Gunn diodes. Finally, we investigate the effect of channel doping which is observed in Fig. 2(b) to be relatively, less influential on the fundamental mode efficiency than the higher harmonic modes, so that for a given notch width and bias voltage, there is an optimum channel doping that favors the harmonic enhancement. However, there is a lower threshold for the channel doping as it determines the dielectric domain nucleation time that needs to be much shorter than the domain transit time through the active region [7].

\section{CONCLUSION}

In summary, we have analyzed the trends in efficiency and harmonic enhancement in GaN Gunn diodes under doping profile, bias and temperature variations. The key parameter in all these efficiency considerations has been the phase angle difference between the current and voltage waveforms. Our extensive simulations indicate that the carrier dynamics in $\mathrm{GaN}$ can be tailored by an optimum choice of doping profile, temperature and bias conditions so that the efficiency of higher harmonic Gunn oscillations can be boosted.

\section{REFERENCES}

1. M. Wraback, H. Shen, J. C. Carrano, T. Li, J. C. Campbell, M. J. Schurman and I. T. Ferguson, Appl. Phys. Lett.76, (2000), 1155.

2. G. M. Dunn and M. J. Kearney, Semicond. Sci. Technol. 18, (2003), 794.

3. C. Sevik and C. Bulutay, Semicond. Sci. Technol. 19, (2004), 188.

4. R. P. Joshi, S. Viswanadha, P. Shah, and R. D. del Rosario, Appl. Phys. 93, (2003), 4836.

5. S. H. Jones, G. B. Tait, and M. Shur, Micowave Opt. Technol. Lett. 5, (1992), 354.

6. R. Judaschke, IEEE Trans. Microwave Theory Tech. 48, (2000), 719.

7. M. Shur, Introduction to Electronic Devices (Wiley, New York, 1996), p. 270. 